\title{
„Handeln statt Hoffen“. Zur Gemeinschaftsanrufung durch Attribution von Verantwortungsgefühlen im medialen Diskurs zur Seenotrettung von Carola Rackete im Sommer 2019
}

\author{
Carola Rackete's Sea Rescue Operation in 2019. A Narrative of Communality \\ Stemming from Feelings of Responsibility
}

\begin{abstract}
ZUSAMMENFASSUNG
Der Beitrag stellt eine Erzählung von Gemeinschaftlichkeit vor, die sich insbesondere aus der Emphase von Gefühlen der Verantwortung speist. Anhand ausgewählter Mediendokumente zur Seenotrettung von Carola Rackete im Sommer 2019 wird dargelegt, unter welchen Umständen eine Gesellschaft ihre Individuen zu gemeinschaftlich verantwortungsvollem Handeln anruft und wann aus der vielfach geäußerten Hoffnung gemeinsames Handeln tatsächlich resultiert. Ein Schwerpunkt liegt auf der Erforschung der Konstitutionsbedingungen von - realen wie auch imaginierten - verantwortlichen Gemeinschaften, also der diskursiven Prozesse der Anrufung zur Gemeinschaft durch die Zuschreibung von Verantwortungsgefühlen. Dabei bedient sich der Beitrag methodologisch Elementen der sprach- und sozialwissenschaftlichen Diskursforschung. Schlüsselwörter: Diskursanalyse, Gefühle, Gemeinschaft, Mediendiskurs, Narrativität, Seenotrettung, Verantwortung
\end{abstract}

\section{ABSTRACT}

The article addresses a narrative of communanlity stemming primarily from feelings of responsibility. Referencing selected media reports on Carola Rackete's sea rescue operation in 2019, the article discusses the circumstances under which a society calls on its individual members to act in a spirit of responsibility for their community - and at which point this common hope actually results in common action. The paper focuses on what constitutes responsible communities real and imagined, i.e. the discursive processes by which a community is invoked through the attribution of feelings of responsibility. The article employs methodological elements from linguistic and social science discourse research.

Keywords: discourse analysis, feelings community, media discourse, narrativity sea rescue, responsibility

Bettina Radeiski, Department Soziale Arbeit, Hochschule für Angewandte Wissenschaften Hamburg, Alexanderstr. 1, 20099 Hamburg, Phone: 004940428757061, bettina.radeiski@haw-hamburg.de, https://orcid.org/0000-0001-6968-5026

Eva Steinmetz, Department Soziale Arbeit, Hochschule für Angewandte Wissenschaften Hamburg, Alexanderstr. 1, 20099 Hamburg, EvaSteinmetz@gmx.de, https://orcid.org/0000-0002-6324-1253 


\section{Einleitung}

Es ist kein Geheimnis, dass die EU-Außengrenzen ein zentrales Konfliktfeld europäischer Politik darstellen - über sie regulieren sich die „Zugangsbedingungen von Menschen zu politischen Räumen“ (Abbasi et al., 2019, S. 9) und damit auch jene öffentlichen Diskurse, welche hierzulande unter den Schlagwörtern „Abschottung“, „Verteilungsfragen“ und „Aufnahmequoten“ kontrovers diskutiert werden. So schien es im Sommer 2019 keineswegs überraschend, dass sich eine mediale Debatte um die damalige Mission des privaten Rettungsschiffes SeaWatch 3 entfachte: Ganze 17 Tage trieben das Schiff, seine Crew und 53 gerettete Menschen auf dem Mittelmeer, da kein geographisch halbwegs nahe gelegenes Mitgliedsland der Europäischen Union die Anlandung ermöglichte - bis die Kapitänin des Schiffes, Carola Rackete, beschloss, trotz eines Verbots der italienischen Regierung in den Hafen von Lampedusa einzufahren.

Während faktisch einmal mehr die Uneinigkeit der Europäischen Union in puncto Seenotrettung sichtbar wurde, ließen sich medial interessanterweise ganz andere Prozesse dokumentieren: So wurde der gesamteuropäische Dissens auf einen Dissens zwischen der Kapitänin Rackete und dem damaligen italienischen Innenminister Matteo Salvini verkürzt, welcher wiederum Einhegung in einen national-gemeinschaftlichen Konsens fand: Salvini wurde in den hiesigen Medien demonstrativ wochenlang als „Unmensch“ beschimpft - Rackete dagegen avancierte zur Symbolfigur des „Helfen-Wollens“ (vgl. Jauernig, 2019) und zur Ikone der „Humanität“, deren Rettungsmission einstimmigen Zuspruch von fast allen Politiker*innen und Medienvertreter*innen der deutschen Presselandschaft verbuchen konnte.

Im vorliegenden Beitrag soll es um diese diskursiv genährte Erzählung von Gemeinschaftlichkeit gehen, die sich - so die These - aus verschiedenen Quellen, insbesondere aber aus der Emphase von Gefühlen der Verantwortung speist. Ziel der Analyse ist es, die Konstitutionsbedingungen von - realen wie auch imaginierten - verantwortlichen Gemeinschaften genauer zu erforschen, also Prozesse der Anrufung zur Gemeinschaft durch die Zuschreibung von Verantwortungsgefühlen in den Blick zu nehmen. Anhand ausgewählter Mediendokumente zur Seenotrettung im Sommer 2019 wollen wir analysieren, unter welchen Umständen eine Gesellschaft ihre Individuen zu gemeinschaftlich verantwortungsvollem Handeln anruft - und wann aus der vielfach geäußerten Hoffnung nach gemeinsamer Verantwortungsübernahme gemeinsames Handeln resultiert.

Dabei gehen wir zunächst davon aus, dass die geäußerten Anrufungen - dies ist ihr erstes und wesentliches Merkmal - uns nicht gleichgültig lassen sollen. Gemeinschaftsanrufungen affizieren (moralisch) - auf die eine oder andere Weise. Alkemeyer und Bröckling (2018) halten solch eine Affektivität sogar für ,unerlässlich“, „um die Einheitsfiktion des Kollektivsubjekts sinnlich zu beglaubigen und im Gefühlsleben aller Beteiligten zu verankern“ (S. 26). Reckwitz (2015) ent- 
wickelt in diesem Zusammenhang den Begriff der „,semiotisch-imaginären Artefakte" und bezeichnet damit "schriftliche Texte und Bilder sowie Kombinationen von beiden“, die als „Affektgeneratoren“ wirken (S. 176). Womit wir bei einem zweiten wesentlichen Merkmal von Gemeinschaftsanrufungen wären: Es gibt sie nicht jenseits dessen, was und wie über Gemeinschaften, ihre Werte, Verhaltensnormen und Gefühlsregeln erzählt wird. Gemeinschaften werden, so die Annahme dieses Beitrags, in erster Linie narrativ, d.h. diskursiv erzeugt.

Beides zusammengenommen bedeutet, dass Gemeinschaften nicht zwangsläufig als ontische Entitäten existieren müssen. Folgt man Andersons Konzept der „,imagined communities“ (Anderson, 2016), so reicht bereits eine ideelle Identifikation mit einer vorgestellten, physisch entfernten Gruppe zur Bildung einer gemeinschaftlichen Identität aus. Zentral seien keineswegs „reale“ Gemeinsamkeiten, sondern allein der Glaube an das Gemeinsame. Dieser Glaube erfordere jedoch eine kontinuierliche Vermittlung von Symbolen - Sprache, Mythen, Geschichten -, die das Konstrukt emotional unterfüttern und Differenz zu anderen erschaffen (vgl. Anderson, 2016).

Diese theoretischen Denkansätze plausibilisieren ein diskursanalytisches Vorgehen, bei dem eine Auswahl von ,semiotisch-imaginären Artefakten“ genauer in den Blick genommen wird.

\section{Das Korpus}

Das Korpus basiert auf 66 in überregionalen Printmedien und deren OnlineAusgaben $^{1}$ veröffentlichten Pressetexten, die u.a. zahlreiche Stellungnahmen unterschiedlicher Politiker*innen enthalten. Rackete veröffentlichte am 4. November 2019 den „Aufruf an die letzte Generation“ mit dem Titel „Handeln statt Hoffen“ (Rackete, 2019) - auch dieses Buch sowie einzelne auf YouTube dokumentierte Interviews wurden in die Analyse einbezogen.

Die Mission der Sea-Watch 3 begann am 12. Juni 2019; es vergingen zwei Wochen, bis die Medien ausführlich über diese berichteten. Mitte Juli ebbte die Aufmerksamkeit bereits wieder ab, die Artikel und Fernsehbeiträge wurden kürzer und seltener. In den Wochen dazwischen wurde das Geschehen von den Medien gewissenhaft verfolgt; Aussagen von Rackete und Salvini wurden von Fachexpert*innen in Interviews mit Pressevertreter*innen und in Gastbeiträgen auf ihre Faktizität hin beurteilt; die Anzahl der Texte, die die Legitimation der Seenotrettung ganz prinzipiell in Frage stellten, kann als gering bezeichnet wer-

1 Es handelt sich um die Ausgaben der Zeitungen Süddeutsche Zeitung bzw. sz.de, Frankfurter Allgemeine Zeitung bzw. faz.net, Die Welt bzw. Welt Online, die Tageszeitung/taz am Wochenende bzw. taz.de sowie Die Zeit bzw. Zeit Online, Der Spiegel bzw. Spiegel Online, Die Tagesschau bzw. tagesschau.de, Der Tagesspiegel bzw. tagesspiegel.de. Die journalistischen Texte wurden im Zeitraum vom 12.6.-31.8.2019 gesichtet. 
den. Die Berichterstattung (v.a. in Form nachempfundener Erlebnisberichte) erfasste den chronologischen Verlauf der Seenotrettung, ihre persönlichen Folgen für Rackete, aber auch den an den verheerenden Zuständen zum Ausdruck kommenden fehlenden politischen Zusammenhalt Europas an seinen Außengrenzen.

Gut zwei Drittel der öffentlichkeitswirksamen Texte entfallen auf kommentierende Texte, zu denen Glossen, Kommentare oder die sogenannte „Seite Drei“ gehören und an deren Ende expressive Meinungskundgaben oder reflektionsorientierte, diskursive Auslegungen stehen. Neben Interviews mit Rackete kamen in unterschiedlichen Beiträgen Unterstützer*innen und Gegner*innen der Seenotrettung zu Wort. Dass Rackete ,trotz Verbots“ Salvinis in den Hafen von Lampedusa einfuhr, wurde zum Anlass genommen, längere oder kürzere Portraits - „Wer ist diese Frau?" (u.a. tagesspiegel.de, taz.de und Der Spiegel) - zu verfassen.

Darüber hinaus ist dem von uns zusammengestellten Korpus eine besondere Charakteristik inhärent: Es zeichnet sich durch eine expansive Hervorbringung und Exposition von Gefühlswissen aus. Dies ist aus zweierlei Gründen nicht überraschend: Gefühlszuschreibungen haben in medial-öffentlichen Debatten in Deutschland ohnehin seit geraumer Zeit Konjunktur. Wie anhand der Korpusanalyse genauer gefasst werden wird, sind Gefühle mittlerweile ein probates Mittel politischen Regierens, denn sie dienen immer häufiger als Legitimationsbasis für politisches (Nicht-)Handeln (vgl. Bargetz \& Sauer, 2010, S. 141). Aber auch aufgrund der virulenten politischen Brisanz des Themas plausibilisieren sich die emotiven Ausuferungen: Weiterhin gilt die Europäische Grenze als ,tödlichste Grenze der Welt" (Pichl \& Tohidipur, 2019, S. 178); die an ihr entstehenden Interessenkonflikte umfassen „hohe menschenrechtliche Implikationen“ (Abbasi et al., 2019, S. 11). Während vordergründig um juristische Fragen gerungen wird, verweisen die anhaltenden Auseinandersetzungen auf grundlegendere, normative Probleme. So konfligieren Bilder von Ertrinkenden mit dem humanitären und philanthropischen Habitus, mit dem sich die Europäische Union seit ihrer Gründung schmückt; nicht zuletzt legen sie die Vulnerabilität einer „souveränen“ und „einigen" EU offen, hinterfragen Illusionen über die Kontrollierbarkeit von Migration und die Herstellbarkeit von ,innerer Sicherheit“, forcieren Überlegungen zur globalen Gerechtigkeit ebenso wie zur kolonialen Vergangenheit und postkolonialen Gegenwart (Abbasi et al., 2019; Friese, 2017).

Kurzum: Die emotive Vereinnahmung kann als Reaktion auf die politisch angespannte Situation im und am Mittelmeer gedeutet werden, zu der sich die Diskursakteure im Diskurs zur Seenotrettung stellen.

\section{Methodisches zur Erfassung moderner Gemeinschaftsanrufung}

Wenn es sprachliche Mittel sind, die Wissen über Gemeinschaft, Werte, Verhaltensnormen und Gefühlsregeln konstituieren und perspektivieren, dann gilt es, diesem Konstitutionsvorgang nachzuspüren und unterschiedliche Perspektiven (inklusive 
den ihnen inhärenten Interessen) zu dechiffrieren (vgl. Felder, 2013a und 2013b). Innerhalb von Diskursen entscheidet sich, welche „Wissensbestände diskursiv weiterbearbeitet werden“ (Felder, 2013a, S. 15) und welche „keine prominente Erwähnung finden“ (S. 15). Wir haben für unsere Analyse die Häufigkeit der Wiederholung in den Blick genommen, da an ihr die Schwere und die Bedeutung des Ereignisses für die Gemeinschaft ablesbar sind. Hierfür wurden die ausgewählten Pressetexte chronologisch gesichtet und jene dominanten sprachlichen Elemente extrahiert, die direkt oder indirekt auf Formen des kollektiven „Wir“ verweisen.

Dabei stellten wir fest: In den unter Punkt 2 genannten Texten ist es der Appell zur Verantwortung, der am häufigsten vorkommt. Es geht um die Verantwortung für das Leben und die Sicherheit der Geretteten, um das Gefühl, Verantwortung übernehmen zu müssen, und die Enttäuschung, dass niemand in Europa - außer natürlich der Crew an Bord - Verantwortung für die in Not geratenen Menschenleben übernommen hat. Verantwortung unterstellt - wie in Punkt 4 zu zeigen sein wird - in ihrer appellativen Funktion Gemeinschaft und zieht ihre Wirkungen vornehmlich aus den angelagerten Gefühlswerten, die im Folgenden an die Affektgeneratoren von Reckwitz (2015) angelehnt werden.

Gefühle steuern Wahrnehmungen, interpretieren Erfahrenes und motivieren Verantwortung. Sie schaffen sich einen gemeinschaftlich-symbolischen Ausdruck, in dem sie zumeist sprachlich materialisiert und/oder gestisch bzw. mimisch visualisiert werden. Damit geht einher, dass die Sichtbarmachung von Emotionen mit ,politisch-kulturellen Wahrnehmungsmuster[n], Normen und sozialen Strukturen" (Bargetz \& Sauer, 2010, S. 143) verquickt ist. Wie wir Gefühle bei uns selbst identifizieren, wie wir sie bei anderen deuten, wie wir sie sprachlich oder mimisch ausdrücken und evozieren - all diese Deutungs- und Definitionsprozesse sind kulturell überformt, historisch erwachsen und gesellschaftlich normiert. Diskursakteure sind also dazu angehalten, Gefühle in adäquater Form zu inszenieren, sodass diese von anderen Gesellschaftsmitgliedern er- und anerkannt werden können. So ist die Tat Racketes nicht nur in Hinblick auf ihren juristisch-politischen Gehalt interessant, sondern auch, wie sie sich dabei fühlt und wo sie die Gemeinschaft implizit aufruft. In einem Spiegel-Interview äußert sich Rackete „wütend und enttäuscht. [...] [W] eder die EU-Kommission noch die Nationalstaaten [sind] bereit, Verantwortung zu übernehmen. Alles muss man selbst machen“ (Rackete zit. nach Lüdke, 2019). Dazu im Folgenden mehr.

\section{Attribution von Gemeinschaftsgefühlen und gemeinsamer Verantwortung - eine exemplarische Analyse}

Das in Kapitel 2 dargestellte Korpus wurde hinsichtlich der unterschiedlichen sprachlichen Formen und Elemente, wie eine Gemeinschaft erzählt wird, und hinsichtlich der vom Diskurs eingesetzten Affektgeneratoren untersucht. Die unterschiedlichen Elemente werden als ein spezifischer Stil des Anrufens betrachtet, 
für den eine affizierende Sprache verpflichtend ist. Im Einzelnen wurden erfasst:

a) das Benennen der für die Narration relevanten Akteure durch:

- das Markieren der politischen Zugehörigkeit (wir, wir Europäer*innen) und typisierende Selbstbezeichnungen (ich als $X$, wir als $X$ ),

- das Etikettieren politischer Rollen (Seenotretter*innen, Politiker*innen, Expert*innen des Seerechts),

- das kontinuierliche Zitieren und Reformulieren von politischen Stellungnahmen,

- das regelmäßige Wiedergeben von Umfrageergebnissen,

- das Sichtbarmachen von Gemeinschaftsbekundungen und -bewegungen (Spendenkonten, Demonstrationen, Kundgebungen, Solidaritätsbekundungen per Hashtag);

b) das Inszenieren der für die Narration relevanten Akteure durch:

- das (teils metakommunikativ problematisierte) Portraitieren einer Heldin, ihren Gegenspielern und Hassfiguren (Glaubwürdigkeit, Authentizität, Inszenierung),

- die Wiedergabe und das Reformulieren von Not, Leid und Verzweiflung der Geflüchteten (Bilder des Schreckens und des Todes),

- das kontinuierliche Widerspiegeln von Gefühlen (Verantwortungsgefühle, Solidaritäts- und Humanitätsgefühle, Solidaritätswellen, Hoffnung, aber auch Enttäuschung, Wut, Schuld, Scham, das Gefühl, alleingelassen worden zu sein)

- das Bekunden von Höchstwerten (Menschenrechte, Menschenleben, Menschlichkeit), für die wir stehen (sollten),

- das Markieren des richtigen Handelns (das Gefühl, das Richtige zu tun) und der Selbstermächtigung,

- das Betonen von moralischen Pflichten;

c) das Bewerten der für die Narration relevanten Akteure durch:

- das Bekunden von Respekt und Hochachtung bzw. Missachtung gegenüber anderen (wer hat tatsächlich „trotz Verbot" Verantwortung übernommen und wer nicht),

- das Loben, Tadeln und Bewerten der eigenen und der fremden Handlungen (wir sind/stehen für Menschenrechte und Menschlichkeit, Europa ist gespalten, rechtsnationalistisch, Gutmenschen),

- das Typisieren, Vergleichen und Gegenüberstellen von Gemeinschaftsmitgliedern (besonnen und ruhig versus aufgebracht und drohend),

- das Skandalisieren der Drangsale der Geflüchteten und damit das Remoralisieren der eigentlich guten Gemeinschaft.

Schauen wir das Material etwas genauer an: Das Gefühl, ,,allein “ mit der Verantwortung für die geretteten Menschen zu sein, zieht sich wie ein roter Faden durch die Pressetexte. Zu Beginn der medialen Debatte vielfach von Rackete 
und der Crew geäußert, zirkuliert es später als retrospektive Momentaufnahme in der Vergangenheitsform durch die deutsche Presselandschaft. Europa lasse die Seenotretter*innen ,im Stich“ (Rackete, zit. nach Lüdke, 2019), die EU-Kommission und die Nationalstaaten ,hätten sich [...] vor ihrer Verantwortung gedrückt" (Rackete, zit. nach Leber, Spannagel, Dembach, Müller, \& Hampel, 2019), sodass der Crew nach 17 Tagen des Ausharrens ,keine andere Wahl“ bleibe, die Menschen an Bord in Sicherheit zu bringen, in letzter Konsequenz auch dann, wenn eine Haftstrafe in Italien drohe.

Teil dieses affektiven Arrangements sind zudem das Vorführen des ,unhaltbaren“ (Rüb, 2019) Notstandes auf dem Schiff sowie die ausführliche Beschreibung der Erlebnisse der Geretteten und deren körperlicher Versehrtheit:

Sie haben Narben auf dem Kopf, sichtbar für jedermann, und unsichtbare Narben auf der Seele [...]. ,Ich bin wahnsinnig wütend, dass es auf der Welt so ungerecht zugeht, also musste ich was tun.' (Rackete und ,Victoria', Ärztin auf der Sea-Watch 3, zit. nach Rackete, 2019, S. 24).

Die zu Wort kommende Ärztin thematisiert ihre „wahnsinnige“ Wut, welche sie zum Handeln motiviert habe. Dabei wird die Ungerechtigkeit der Welt qua Versprachlichung und Bebilderung des Leidens der Geretteten inszeniert, welche im ganzen Diskursausschnitt die Funktion eines affektiven Katalysators einnimmt: Die Exposition des Leidens „der Anderen“ ebnet vielfach den Übergang zur Anrufung einer gemeinschaftlichen/singulären Verantwortungsübernahme. Friese stellt in diesem Kontext fest, dass sich Seenotrettungsdiskurse durch ein massenmediales ,public viewing des Schreckens“"2 (Friese, 2017, S. 57) auszeichneten, welches die Rezipient*innen anhalte, affektiv auf das Wahrgenommene zu reagieren. Die mediale Verarbeitung der Bild- und Gefühlswelten fordere dazu auf, dass ,politische Verantwortung zu übernehmen [sei], in die ,wir‘ eingebunden sind, weil wir auf die Not der anderen antworten müssen und daher Verantwortung zu übernehmen haben“" (S. 94).

Friese zufolge verbindet sich das „Wir“ über Prozesse der Selbst- und Fremdzuschreibung von Verantwortung, die - wie, Victoria' im obigen Zitat verdeutlicht durch Gefühle, hier negative (,wahnsinnig wütend“), dynamisiert werden können. Ohnehin spielen Gefühle wie Wut, Enttäuschung und Verzweiflung im untersuchten Diskursausschnitt eine dominante Rolle; mit ihnen werden der Unmut über das „Nichthandeln der politischen Verantwortlichen“ (Leber et al., 2019) verbalisiert und entsprechende Vorwürfe und Klagen an die Entscheidungsträger*innen

2 Friese zeigt auf, dass die mediale Aufmerksamkeitsökonomie kontinuierlich Bilder von nackten, schonungslos offengelegten - zumeist schwarzen - Körpern zeige, die hilflos mit dem Tode rängen (vgl. Friese, 2017, S. 57-59). Damit würden die dargestellten Menschen in einer ,unendlich andauernden Opferposition“ (S. 92) fixiert, die ihnen jegliche Handlungsmöglichkeit und Subjektivität abspreche. Erst durch diese Festschreibung des Opferstatus ließen sich die heldenhaften Rettungsaktionen kontrastierend und massenwirksam legitimieren. 
adressiert. Interessanterweise steckt jedoch auch in diesen Gefühlen ein impliziter Gemeinschaftsgedanke: Durch die wiederholte Emphase von Enttäuschung und Wut über das Ausbleiben einer dringend indizierten und praktizierten Lösung für die Geretteten konstelliert sich auf indirektem Wege eine ,imagined community“. Die Klage, „im Stich“ oder „alleingelassen zu werden“, macht schließlich nur dann Sinn, wenn ein - wie auch immer - antizipierter Gemeinschaftsgedanke unterstellt wird, mit dem Erwartungen und Hoffnungen verknüpft sind.

Dabei lässt der Diskurs keinerlei Zweifel daran, welches Narrativ eines gemeinschaftlichen Europas gehegt und gepflegt werden soll: Spätestens mit der Einfahrt der Sea-Watch 3 in den Hafen von Lampedusa und Racketes darauffolgender Verhaftung meldeten sich allerlei Politiker*innen und Pressevertreter*innen zu Wort, für die ,indiskutabel“ und ,selbstverständlich“ feststand, dass „Menschenleben $[\ldots]$ auf jeden Fall und egal auf welche Weise gerettet werden [müssten]“ (Kardinalstaatssekretär Pietro Parolin zit. nach bard./dpa, 2019). Schnell war von einem Skandal die Rede, gar von ,weltweiter Empörung“ (Grasnick \& Culina, 2019). Aus der Skandalforschung ist hinreichend bekannt, dass die verdichtete Vorführung ausgewählter Emotionen - vorzugsweise jener der Entrüstung und Empörung - dazu dient, einen von der Gesellschaft identifizierten Missstand zu problematisieren und diesen als Normbruch zu markieren: Folglich fungiere der Medienskandal zur „Produktion von Normalität“ (Pundt, 2008, S. 220); er bringe (vermeintlich) konsensual geteilte Werte und Normen auf die öffentliche Agenda und fordere deren Geltung ein.

Remoralisiert werden soll in unserem Falle die ,gute Gemeinschaft", die sich (wieder) zu sogenannten „europäischen“ Werten, höchsten Werten bekennt, indem sie humanitär handelnd auftritt und so ihren propagierten Werten entspricht. So schreibt Vahland in einem längeren Portrait in der Süddeutschen Zeitung, dass Rackete in den Augen ihrer Unterstützer*innen ,antr[ete], die europäischen und christlichen Werte gegen den mit Menschenverachtung prahlenden italienischen Innenminister Matteo Salvini durchzusetzen“ (Vahland, 2019). Grahovac (2019) kommentiert in der taz am Wochenende die Situation wie folgt:

Ja, das ist Europa in diesen Zeiten: Es lässt nicht nur massenhaft und immer wieder Menschen auf jenem Meer ertrinken, [...] es bringt diejenigen in Gefahr, die den Humanismus, auf dem die EU gründet, verinnerlicht haben und leben. Als sei irgendwo auf dem Meer eine Grenze gezogen worden, hinter der die Werte, auf denen die Staatengemeinschaft fußt, nicht gelten: die Wahrung der Menschenrechte, Hilfe in Not.

Konzeptionalisiert wird in diesem Zitat ein idealisiertes Innen - eine Staatengemeinschaft, welche Menschen wie Rackete hervorbringe, die „den Humanismus [...] verinnerlicht haben und leben“ - im Gegensatz zu einem territorial abgegrenzten Außen, wo die in zahlreichen Deklarationen kodifizierten Werte ihre Geltungskraft verlören. Sogar Horst Seehofer, bekannt als Hardliner in puncto 
Geflüchtetenpolitik, möchte Teil dieser erhabenen Formation werden und moniert in einem vielzitierten Brief an Salvini, dass es wegen der gemeinsamen europäischen Verantwortung ,und unseren gemeinsamen christlichen Werten [...] im Einzelfall keinen Unterschied machen [dürfe], durch welche Organisation Migranten aus dem Mittelmeer gerettet wurden" (Seehofer, zit. nach Franke, 2019).

Ungeachtet der Tatsache, dass in den hier ausgewählten Zitaten unterschiedliche Vorgänge problematisiert werden - Salvinis Hasstiraden, das Mittelmeer als Todesort, die Uneinigkeit der europäischen Staaten bei der Aufnahme der geretteten Menschen - wird in allen drei Ausschnitten ein loser Bezug zu ,gemeinsamen europäischen Werten“ hergestellt, für die wir - als Europäer*innen - eigentlich stehen sollten. Als moralischer Referenzrahmen werden „Humanismus“, „Menschenrechte“ und „Hilfe in Not“ reklamiert, in anderen Pressetexten wird „Europa“ zudem zum Hort der „Demokratie“, „Gleichheit" und „Freiheit" stilisiert. Allein die Beschwörung von Humanität lässt sich dutzendfach aus den vorliegenden Texten herausfiltern. Die rekurrenten Darstellungen dieser Abstrakta lassen darauf schließen, dass die Diskursteilnehmer*innen diesen normativen Selbstbeschreibungen Gewicht zukommen lassen wollen; letztendlich konsolidiert sich auch damit ein „Wir“, das auf der intersubjektiv hergestellten Vergewisserung basiert, an der Vorstellung einer europäischen Wertegemeinschaft festzuhalten, trotz - oder gerade wegen - der Enttäuschung über deren ausbleibende empirische Wirkmächtigkeit.

Nun sind Hochwertwörter wie „Humanismus“ und „Freiheit“ als abstrakte Entitäten zu verstehen, deren Beschwörung vielmehr ein symbolisch-affektiver Gehalt als eine konkrete empirische Figuration inhärent ist. Gewissermaßen stehen Hochwertwörter ,über der aktuellen politischen Diskussion“ (Wengeler, 2017, S. 26), da sie nicht parteilich-programmatisch gebunden sind und damit gerne und häufig von allen Diskursakteuren - unabhängig von deren politischen Einstellungen - in Anschlag gebracht werden. Die Beschwörung von Humanität geht - salopp gesagt - auch deshalb so leicht von der Zunge, weil Hochwertwörter fast ausnahmslos auf Konzepte verweisen, die gesellschaftlich unumstritten und positiv konnotiert sind. Mit ihnen lässt sich die eigene Diskursposition mit einem ,positiven Glanz“ (Wengeler, 2017, S. 31) versehen, sodass Diskursakteure um die Besetzung und Aneignung der Wörter ringen. Dabei zeigt sich: Je höher der beschworene Wert, desto leichter lässt sich damit Zustimmung generieren. Wer würde schon Bundesaußenminister Heiko Maas widersprechen, wenn er auf Twitter von der humanitären Pflicht, Menschenleben zu retten, spricht (vgl. Maas, 2019)?

Völlig unterbelichtet bleibt jedoch die Tatsache, dass neben der konsensual-fixierten, deontischen Komponente der Begriffe auf der semantischen Bestimmungsebene keinesfalls Einigkeit darüber herrscht, wie die konkrete Erscheinungsform von Humanität auszusehen habe. Angesichts der Kontinuität der verheerenden Zu- 
stände an den europäischen Außengrenzen (inklusive Verschiebungen des Grenzregimes nach Libyen und in die Türkei) und den damit verbundenen politischen Streitigkeiten überrascht es nicht, dass die Medienvertreter*innen keinesfalls die semantische, sondern schlicht die appellative Bedeutungskomponente der Werte fixieren: Die appellative Funktion von „Humanität" und „Gleichheit" tritt einmal mehr in den Vordergrund und kanalisiert sich im Ruf nach Verantwortung, die - von wem auch immer, oft von „,der gesamten EU“3 - zu übernehmen sei. Die komplexe Frage verengt sich damit auf die Rede von Verantwortlichkeit.

Verstärkt und flankiert wird die kontinuierliche Anrufung zum gemeinsamen verantwortungsvollen Handeln durch die Vorführung gelingender Gemeinschaftlichkeit in Form von Privatinitiativen, welchen diskursiv ein dominanter Platz im medialen Geschehen um die Sea-Watch 3 eingeräumt wird. Es mag als ein Teilerfolg diskursiver Anrufungen gelten, dass einzelne Individuen sich vom ausgerufenen „Notstand der Menschlichkeit“" (Demonstrationsparole, zit. nach Schipkowski, 2019) angesprochen fühlen und daraufhin handeln. Die Initialzündung hierfür lieferten die Fernsehmoderatoren Jan Böhmermann und Klaas Heufer-Umlauf, deren Spendenaufruf nach der Verhaftung Racketes binnen weniger Stunden viral ging und horrende Summen im 7-stelligen Bereich für die Hilfsorganisation einbrachte. Da die Moderatoren - wie die meisten - nicht persönlich vor Ort helfen könnten, ,möchten wir spenden und gemeinsam mit Euch Geld sammeln“ (Gemeinsame Erklärung zur Spendenaktion zit. nach bard./dpa, 2019). „Ihr könnt mitmachen. Und man steht nicht ganz tatenlos daneben“" (Heufer-Umlauf \& Böhmermann, 2019).

Die hier bemühten Begrifflichkeiten lassen zweifelsohne durchscheinen, dass erstens, die mehrheitlich vorhandene Unfähigkeit, persönlich vor Ort aktiv zu werden, durch den Klick auf einen Spendenlink neutralisiert werden könne und zweitens, dass gemeinsames Handeln - mit-machen - wirksam - da nicht tatenlos - sei. Unterfüttert wird das anklingende Machbarkeitsnarrativ in den folgenden Tagen der Berichterstattung durch unzählige Verweise auf die Höhe der eingegangenen Spenden, auf die Anzahl der Spender*innen sowie auf andere Initiativen, die sich mit den Seenotretter*innen solidarisierten: Medial aufgegriffen wurden unter anderem der Hashtag \#FreeCarola sowie zahlreiche, von der Organisation Seebrücke initiierte Demonstrationen in unterschiedlichen Städten, darunter „[s]ogar New York“ (Schipkowski, 2019).

In all diesen Solidaritätsbekundungen manifestiert sich die Anrufung zur Gemeinschaft mittels der Vorführung, dass und wie gemeinschaftliches Handeln funktionieren kann; sie symbolisieren das Aufscheinen des Möglichen und stehen deshalb im affektiven Diskursarrangement für Gefühle der Solidarität, Zu-

3 Auch hier gilt das Prinzip: Je höher, also allgemeiner und abstrakter das angerufene Subjekt, desto unwidersprechlicher erscheint der Aufruf. 
gehörigkeit und Hoffnung. Eng verknüpft sind diese „Wir“-Konstruktionen mit unterschiedlichen Strategien der moralischen Absicherung und Einhegung: Wenn „Sogar" eine Stadt auf der anderen Seite des Ozeans der Causa Rackete Beachtung schenke, bestätigt sich die Wichtigkeit und Richtigkeit des gemeinschaftlichen Aktionismus. Zahlreiche Verweise auf die Masse der Demonstrant*innen (,so viele“) und auf die Wucht der artikulierten Solidarität („Spenden-, Solidaritäts-, Sympathiewelle") kolorieren jenes Bild, welches qua ARD-Umfrage dann sogar empirisch validiert wird. So kommt die am 4. Juli 2019 erstmals publizierte - und später vielfach rezitierte - Befragung zu dem Schluss, dass ,drei Viertel der Deutschen [...] private Seenotretter gut [fänden]“ (EPD-BAS, 2019).

Die in Anschlag gebrachten Superlative zur Beschreibung der sich formierenden Gemeinschaft, verquickt mit dem immer wieder anklingenden Motiv, „das Richtige zu tun“, evoziere bei den Leser*innen den Eindruck, „Teil einer globalen Bewegung gegen die Flüchtlingspolitik der EU“ (Lobenstein, 2019) zu sein. Der an anderer Stelle explizierte Ruf nach politischer Verantwortung wird also diskursiv aufgegriffen und das sichtbargemachte private Engagement so drapiert, als würde es selbst das leisten, was es einfordert: die Wahrnehmung der Verantwortung durch die politisch zuständigen Instanzen. Indem sich auch Politiker*innen mittels ausschweifender Solidaritätsbekundungen in das gemeinschaftliche Narrativ einschreiben, ist die Überschattung der politisch prekären Ausgangslage de facto schon realisiert:

Die Lebensretter auf See dürfen nicht kriminalisiert werden. [...] Das menschliche Drama im Mittelmeer verhindern wir, indem wir Fluchtursachen bekämpfen und nicht wegsehen, wenn sich Menschen auf den lebensgefährlichen Weg in einen sicheren Hafen machen (Malu Dreyer, zit. nach dpa/FAZ.NET, 2019).

Der Schulterschluss mit den Seenotretter*innen vollzieht sich durch die Synthese zum überinstitutionellen Wir, das nicht mehr zwischen Privatinitiative und politischer Zuständigkeit trennt.

\section{Fazit}

Zwar sind die sprachlich manifestierten Emotionen im Sommer 2019 äußerst variant - alles, was einigermaßen dramatisch anmutet, ist im Material vertreten -, sie laufen aber kontinuierlich in einem Punkt zusammen. „Enttäuschung“, „Angst“, „Verzweiflung“ ebenso wie „Mut“, „Hoffnung“ und „Stolz“ werden vom Diskurs derart synthetisiert, dass sie letztlich zum Ausdruck eines singulären Gefühlkonsenses werden: In allen betreffenden Textstellen wird eine gemeinsame Verantwortung unterstellt oder an diese appelliert - wobei es im Sinne Felders keineswegs überrascht, dass unterschiedliche Objekte und Formen der Verantwortung je nach Sprecher*innenposition identifiziert werden können. Ein kollektives „Wir“ formierte sich keineswegs auf der Ebene der politischen Inhalte, 
sondern durch die kontinuierliche Hervorbringung emotiver Deutungsschemata. Gefühlt sind sich dann doch alle Beteiligten einig; so einig, dass vormals völlig disparate politische „Bemühungen“-z. B. die Abschaffung jeglicher staatlicher Seenotrettung - offenbar kurzerhand in den Raum des Vergessens gedrängt und durch den einstimmigen Lobgesang auf Rackete übertönt wurden: Politiker*innen avancierten zu Unterstützer*innen ihrer eigenen Kritiker*innen, indem sie sich - unter Berufung auf Humanität und Menschenrechte - ins real handelnde „Wir“ einschrieben. Damit verschiebt sich der Diskurs von der politisch höchst umstrittenen Frage nach politischer Zuständigkeit hin zur zum Teil geradezu märchenhaft anmutenden Erzählung von Held*innen und ihren Gegner*innen: Ein Einigkeitsnarrativ, das jede*n von uns ansprechen und affizieren soll. Letztlich wurde die Kette der Verantwortlichkeit so konstruiert, dass sie am Ende von der tapferen Kapitänin über engagierte Unterstützer*innen, Spender*innen und Follower*innen bis zum*zur medial informierten Adressat*in herunterreicht. Wir alle sollten uns als mitbestimmende Subjekte einer (humanitären) Gemeinschaft verstehen. Allerdings: Die Gemeinschaft auf ihre Mitverantwortung hin anzurufen, entlastet die wirklich Zuständigen von deren Verantwortung. Wenn sie auf ihrem besonderen Posten als Mit-Verantwortliche angesprochen werden, werden sie als Teilmenge einer imaginären, insgesamt verantwortlichen Gemeinschaft angerufen, in der ein*e jede*r an seinem*ihrem Platze das seine*ihre beizutragen habe. Es handelt sich hierbei um eine Technik der Veredelung sowohl der politisch Verantwortlichen wie des Gemeinwesens, welchem sie vorstehen. Anrufungen zu „Humanität“ und zu „Solidarität“ sprechen das Individuum auf seinen ,privaten“ Beitrag an; als Versuche, die wirklich Zuständigen auf die politische Verantwortung für ihre Taten und Unterlassungen festzulegen, sind diese Dokumente des privaten Gewissens Dokumente der Ohnmacht.

An der Debatte um Rackete und die Sea-Watch 3 zeigt sich, wie leicht es ist, mittels emotiver Ausuferungen Gemeinschaft diskursiv anzurufen und diese zu antizipieren - Verantwortung als ubiquitäre Zuschreibung und moralisch hoch aufgeladenes Programm scheint sich hierfür im Besonderen anzubieten. Gleichzeitig macht die Kontinuität der katastrophalen Zustände an den europäischen Außengrenzen deutlich: $\mathrm{Ob}$ aus den hoffnungsvoll angerufenen Gemeinschaften real handelnde Kollektive werden, steht auf einem völlig anderen Blatt.

\section{References}

Abbasi, S., Hochleitner, A., Kania, M., Känner, L., Kirsch, T., Imgarten, N., Inagaki, K., Kaptan, T., Pichl, M., Tohidipur, T., \& Ugrin, M. (2019). Grenzbetrachtungen. In M. Pichl, \& T. Tohidipur (Eds.), An den Grenzen Europas und des Rechts. Interdisziplinäre Perspektiven auf Migration, Grenzen und Recht (pp. 9-16). Bielefeld: transcript.

Alkemeyer, T., \& Bröckling, U. (2018). Jenseits des Individuums. Zur Subjektivierung kollektiver Subjekte. Ein Forschungsprogramm. In T. Alkemeyer, U. Bröckling, \& T. Peter (Eds.), Jenseits der Person. Zur Subjektivierung von Kollektiven (pp. 17-31). Bielefeld: transcript. 
Anderson, B. (2016). Imagined Communities: Reflections on the Origin and Spread of Nationalism. London, New York: Verso.

bard./dpa. (2019, June 30). Kapitänin Rackete verteidigt Einfahrt in Hafen. FAZ.NET. Retrieved December 1, 2020, from https://www.faz.net/aktuell/politik/boehmermann-und-heufer-umlaufspenden-fuer-rackete-von-sea-watch-16261154.html.

Bargetz, B., \& Sauer, B. (2010). Politik, Emotionen und die Transformation des Politischen. Eine feministisch-machtkritische Perspektive. Österreichische Zeitschrift für Politikwissenschaft, 2(39), 141-155.

dpa/FAZ.NET. (2019, June 30). Bundespräsident kritisiert Festnahme von Sea-Watch-Kapitänin. FAZ.NET. Retrieved December 1, 2020, from https://www.faz.net/aktuell/politik/inland/festnahme-von-carola-rackete-steinmeier-verteidigt-sea-watch-kapitaenin-16261654.html.

EPD-BAS. (2019, July 5). Knapp drei Viertel der Deutschen finden private Seenotretter gut. FAZ. NET. Retrieved December 1, 2020, from https://www.faz.net/aktuell/politik/inland/seenotretter-haben-in-deutschland-starken-rueckhalt-16269794.html.

Felder, E. (2013a). Faktizitätsherstellung mittels handlungsleitender Konzepte und agonaler Zentren. Der diskursive Wettkampf um Geltungsansprüche. In E. Felder (Ed.), Faktizitätsherstellung in Diskursen. Die Macht des Deklarativen (1st ed.) (pp. 13-18). Berlin, Boston: De Gruyter.

Felder, E. (2013b). Linguistische Diskursanalyse im Forschungsnetzwerk Sprache und Wissen. In W. Viehöver, R. Keller, \& W. Schneider (Eds.), Diskurs - Sprache - Wissen. Interdisziplinäre Beiträge zum Verhältnis von Sprache und Wissen in der Diskursforschung (pp. 167-198). Wiesbaden: Springer.

Franke, M. (2019, July 7). Kurz unterstützt Salvini im Streit um Hafenöffnung. FAZ.NET. Retrieved December 1, 2020, from https://www.faz.net/aktuell/politik/ausland/streit-um-hafenoeffnungkurz-unterstuetzt-salvini-16271624.html.

Friese, H. (2017). Flüchtlinge: Opfer - Bedrohung - Helden. Zur politischen Imagination des Fremden. Bielefeld: transcript.

Grahovac, E. (2019, July 6). Rackete hat es uns allen zu leicht gemacht. taz am Wochenende, 19.

Grasnick, B., \& Culina, K. (2019, July 1). Lebensretterin steht unter Hausarrest. taz, 3.

Heufer-Umlauf, K., \& Böhmermann, J. (2019, July 1). Menschenleben retten ist kein Verbrechen! Spendenaufruf für Carola Rackete von Sea Watch [Video]. YouTube. Retrieved December 1, 2020, from https://www.youtube.com/watch?v=8Dc0xibgwSE.

Jauernig, H. (2019, July 13). Salvini wirft Frankreich Heuchelei vor. Spiegel Online. Retrieved December 1, 2020, from https://www.spiegel.de/politik/ausland/carola-rackete-erhaelt-preisin-frankreich-matteo-salvini-spricht-von-heuchelei-a-1277204.html.

Leber, S., Spannagel, L., Dernbach, A., Müller, K., \& Hampel, T. (2019, July 3). Wer ist Carola Rackete? Das erzählen Weggefährten über die „Sea-Watch“-Kapitänin. Der Tagesspiegel. Retrieved December 1, 2020, from https:/www.tagesspiegel.de/politik/wer-ist-carola-racketedas-erzaehlen-weggefaehrten-ueber-die-sea-watch-kapitaenin/24517714.html.

Lüdke, S. (2019, June 26). „Sea-Watch 3” steuert trotz Verbot Hafen von Lampedusa an. Spiegel Online. Retrieved December 1, 2020, from https://www.spiegel.de/politik/ausland/sea-watch-3-kapitaenin-carola-rackete-im-interview-bereit-die-konsequenzen-zu-tragen-a-1274507. html.

Maas, H. (2019, June 20). Menschenleben zu retten ist eine humanitäre Verpflichtung. Seenotrettung darf nicht kriminalisiert werden. Es ist an der italienischen Justiz, die Vorwürfe [Tweet]. Retrieved December 1, 2020, from https://twitter.com/HeikoMaas/status/1144949474076319744.

Pichl, M., \& Tohidipur, T. (2019). Verpflichtung zur aktiven Seenotrettung. Zu den nicht intendierten Effekten der Überwachung des Mittelmeers durch Frontex. In M. Pichl, \& T. Tohidipur (Eds.), An den Grenzen Europas und des Rechts. Interdisziplinäre Perspektiven auf Migration, Grenzen und Recht (pp. 177-210). Bielefeld: transcript. 
Pundt, C. (2008). Medien und Diskurs. Zur Skandalisierung von Privatheit in der Geschichte des Fernsehens. Bielefeld: transcript.

Rackete, C. (2019). Handeln statt Hoffen. Aufruf an die letzte Generation. München: Droemer.

Reckwitz, A. (2015). Praktiken und ihre Affekte. Mittelweg 36, 24(1/2), 27-45.

Rüb, M. (2019, June 30). Riskantes Manöver und unerwünschte Belehrungen. FAZ.NET. Retrieved December 1, 2020, from https://www.faz.net/aktuell/politik/ausland/sea-watch-kapitaeninrackete-spaltet-das-politische-italien-16262015.html.

Schipkowski, K. (2019, Juli 5). Seebrücke ist überall. Taz, 6.

Vahland, K. (2019, Juli 2). Im Sog der Idealistin. Süddeutsche Zeitung, 4.

Wengeler, M. (2017). Wortschatz I: Schlagwörter, politische Leitvokabeln und der Streit um Worte. In K. S. Roth, M. Wengeler, \& A. Ziem (Eds.), Handbuch Sprache in Politik und Gesellschaft (pp. 22-46). Berlin, Boston: De Gruyter. 